Proc. Indian Acad. Sci. (Math. Sci.) Vol. 113, No. 4, November 2003, pp. 443-449.

Printed in India

\title{
Uniform stability of damped nonlinear vibrations of an elastic string
}

\author{
GANESH C GORAIN and SUJIT K BOSE* \\ Department of Mathematics, J.K. College, Purulia 723 101, India \\ *BE 188, Salt Lake City, Kolkata 700 064, India \\ Email: sujitkbose@yahoo.com
}

MS received 21 April 2003; revised 5 August 2003

\begin{abstract}
Here we are concerned about uniform stability of damped nonlinear transverse vibrations of an elastic string fixed at its two ends. The vibrations governed by nonlinear integro-differential equation of Kirchoff type, is shown to possess energy uniformly bounded by exponentially decaying function of time. The result is achieved by considering an energy-like Lyapunov functional for the system.
\end{abstract}

Keywords. Uniform stability; Kirchoff type wave equation; Lyapunov functional; exponential energy decay.

\section{Introduction}

The textbook treatment of transverse vibrations of a metallic wire (string), governed by the linear wave equation, does not portray existence of whirling out of plane motion. The phenomenon was first observed by Hunton as reported by Harrison [9]. The reference to other subsequent experiments can be found in the book by Nayfeh and Mook [14]. It is observed that the whirling motion, sometimes also referred to as ballooning motion occurs when the amplitude and frequency of a plane excitation and the phase difference between the response and the excitation exceeds certain critical values. The explanation of the phenomenon lies in a nonlinear treatment of the problem. Long ago Kirchoff [10], taking longitudinal elasticity also into consideration, gave the following nonlinear integrodifferential equation for transverse vibrations confined to a plane:

$$
\frac{\partial^{2} u}{\partial t^{2}}+2 \delta \frac{\partial u}{\partial t}=\left[a^{2}+b \int_{0}^{l}\left|\frac{\partial u}{\partial x}\right|^{2} \mathrm{~d} x\right] \frac{\partial^{2} u}{\partial x^{2}}, \quad 0<x<l, \quad t \geq 0
$$

where $u(x, t)$ is the transverse deflection, $l$ the length of the string, $\delta>0$ the coefficient of damping, $a^{2}=T_{0} / \rho A$ and $b=E / 2 \rho l$. The constants $T_{0}, \rho, A, E$ are respectively the initial axial tension, the mass density, the cross-section area and Young's modulus of the string. For treating nonlinear out of plane motion with components $(v, w)$ in the perpendicular $y$ and $z$ directions, Anand [1] derives a generalization of equation (1a):

$$
\frac{\partial^{2} \mathbf{u}}{\partial t^{2}}+2 \delta \frac{\partial \mathbf{u}}{\partial t}=\left[a^{2}+b \int_{0}^{l}\left|\frac{\partial \mathbf{u}}{\partial x}\right|^{2} \mathrm{~d} x\right] \frac{\partial^{2} \mathbf{u}}{\partial x^{2}}, \quad 0<x<l, \quad t \geq 0
$$


where $\mathbf{u}$ has components $(v, w)$. A systematic rederivation of (1b) can also be found in Nayfeh and Mook [14]. The book [14] also refers to other authors who derived different nonlinear differential equations under various assumptions.

For a string fixed at both ends, we have

$$
\mathbf{u}(0, t)=\mathbf{u}(l, t)=0
$$

and if $\mathbf{u}_{0}(x), \mathbf{u}_{1}(x)$ are initial displacement and velocity

$$
\mathbf{u}(x, 0)=\mathbf{u}_{0}(x), \quad \frac{\partial \mathbf{u}}{\partial t}(x, 0)=\mathbf{u}_{1}(x), \quad 0<x<l .
$$

The existence and uniqueness in the scalar case are discussed in [12] and [15].

Arosio and Spagnola [3], Gough [7] and several other authors referred to in Nayfeh and Mook [14] have studied the problem $(1 \mathrm{a}, \mathrm{b})$ from mathematical and physical points of view with a focus on modal analysis and eigenvalues of the system. In particular, Anand [2] has investigated (1b) proving that the vibrations are stable even in the planar case. The elaborate method of treatment employs stability analysis of the time-dependent Hill's equation satisfied by the modal amplitudes. In recent years, Shahruz [16] has treated the problem (1a) with a bounded input disturbance and his result shows boundedness of the ouput. The approach is systems and control theoretic.

The mathematical theory of stabilisation of distributed parameter systems is currently of interest in view of application to vibration control of various structural elements. The most common class of vibration control mechanism is of passive type that absorbs vibration energy. A system is called strongly stable if the total energy $E(t)$ of each solution of the system converges to zero as time $t \rightarrow+\infty$. If the convergence is uniform for $t>0$ with respect to all initial data in the energy space for which $E(0)<\infty$, the system is called uniformly stable. Here our investigation is a direct Lyapunov stability approach to obtain uniform exponential energy decay estimate for the system (1b), (2), (3) and establish that the system is a passive energy absorber. Such estimate has earlier been obtained by Gorain [4] for damped linear wave equation in a bounded domain in $R^{n}$. Gorain and Bose [56] have obtained such estimates for an internally damped beam governed by linear equations in torsional and flexural modes of vibration.

\section{Energy of the system}

The total energy at time $t$, of the system (1b)-(3) is defined by the functional

$$
E(t)=\frac{1}{2} \int_{0}^{l}\left[\left|\frac{\partial \mathbf{u}}{\partial t}\right|^{2}+a^{2}\left|\frac{\partial \mathbf{u}}{\partial x}\right|^{2}\right] \mathrm{d} x+\frac{b}{4}\left[\int_{0}^{l}\left|\frac{\partial \mathbf{u}}{\partial x}\right|^{2} \mathrm{~d} x\right]^{2}, \quad t \geq 0
$$

Diferentiating (4) with respect to $t$ and using the governing equation (1b) we obtain

$$
\begin{aligned}
\frac{\mathrm{d} E}{\mathrm{~d} t}= & \int_{0}^{l} \frac{\partial \mathbf{u}}{\partial t} \cdot\left[\left(a^{2}+b \int_{0}^{l}\left|\frac{\partial \mathbf{u}}{\partial x}\right|^{2} \mathrm{~d} x\right) \frac{\partial^{2} \mathbf{u}}{\partial x^{2}}-2 \delta \frac{\partial \mathbf{u}}{\partial t}\right] \mathrm{d} x \\
& +\left(a^{2}+b \int_{0}^{l}\left|\frac{\partial \mathbf{u}}{\partial x}\right|^{2} \mathrm{~d} x\right) \int_{0}^{l} \frac{\partial \mathbf{u}}{\partial x} \cdot \frac{\partial^{2} \mathbf{u}}{\partial x \partial t} \mathrm{~d} x \\
= & \left(a^{2}+b \int_{0}^{l}\left|\frac{\partial \mathbf{u}}{\partial x}\right|^{2} \mathrm{~d} x\right) \int_{0}^{l} \frac{\partial}{\partial x}\left(\frac{\partial \mathbf{u}}{\partial t} \cdot \frac{\partial \mathbf{u}}{\partial x}\right) \mathrm{d} x-2 \delta \int_{0}^{l}\left|\frac{\partial \mathbf{u}}{\partial t}\right|^{2} \mathrm{~d} x
\end{aligned}
$$


The value of the first integral vanishes in view of the boundary conditions (2). We thus get

$$
\frac{\mathrm{d} E}{\mathrm{~d} t}=-2 \delta \int_{0}^{l}\left|\frac{\partial \mathbf{u}}{\partial t}\right|^{2} \mathrm{~d} x, \quad t \geq 0
$$

The negativity of the right hand side of (5) shows that the energy $E(t)$ is decreasing with time due to the incorporation of passive viscous damping coefficient $\delta>0$. The system is thus non-energy conserving. Naturally the question arises as to whether the system is uniformly stable or not. An affirmative answer is contained in the following theorem, which essentially states that in fact the energy of vibration has uniform exponential decay.

Theorem. Let $\mathbf{u}(x, t)$ be a solution of the system (1b)-(3) with $\mathbf{u}_{0} \in H^{1}[0, l]$ and $\mathbf{u}_{1} \in$ $L^{2}[0, l]$, where $H^{1}[0, l]$ is the Sobolev space of order 1 , then the energy $E(t)$ of the system defined by (4) satisfies

$$
E(t) \leq M \mathrm{e}^{-\mu t} E(0), \quad t>0
$$

for some reals $\mu>0$ and $M>1$.

From eq. (4)

$$
E(0)=\frac{1}{2} \int_{0}^{l}\left[\left|\mathbf{u}_{1}\right|^{2}+a^{2}\left|\frac{\mathrm{d} \mathbf{u}_{0}}{\mathrm{~d} x}\right|^{2}\right] \mathrm{d} x+\frac{b}{4}\left[\int_{0}^{l}\left|\frac{\mathrm{d} \mathbf{u}_{0}}{\mathrm{~d} x}\right|^{2} \mathrm{~d} x\right]^{2} .
$$

From eq. (6) it follows that if $E(0)<\infty$ with respect to all initial values $\mathbf{u}_{0} \in H^{1}[0, l]$ and $\mathbf{u}_{1} \in L^{2}[0, l]$ then $E(t) \rightarrow 0$ as $t \rightarrow \infty$ and the system is uniformly stable. In order to prove (6), let $\varepsilon>0$ be a fixed constant. Proceeding as in Gorain [4] (see also Komornik [11]), we define an energy-like Lyapunov functional $V$ according to

$$
V(t)=E(t)+\varepsilon G(t),
$$

where

$$
G(t)=\int_{0}^{l}\left(\mathbf{u} \cdot \frac{\partial \mathbf{u}}{\partial t}+\delta|\mathbf{u}|^{2}\right) \mathrm{d} x
$$

Now since $\mathbf{u}(0, t)=\mathbf{0}=\mathbf{u}(l, t)$, the Poincaré-like Scheefer's inequality

$$
\int_{0}^{l}|\mathbf{u}|^{2} \mathrm{~d} x \leq \frac{l^{2}}{\pi^{2}} \int_{0}^{l}\left|\frac{\partial \mathbf{u}}{\partial x}\right|^{2} \mathrm{~d} x, \quad t>0
$$

holds (see [13] and [8]). A simple proof is provided in the Appendix. Hence using Schwarz's inequality, we obtain

$$
\begin{aligned}
\left|\int_{0}^{l} \mathbf{u} \cdot \frac{\partial \mathbf{u}}{\partial t} \mathrm{~d} x\right| & \leq \frac{l}{\pi a} \int_{0}^{l}\left|\frac{\partial \mathbf{u}}{\partial t}\right|\left|\frac{\pi a}{l} \mathbf{u}\right| \mathrm{d} x \\
& \leq \frac{1}{2} \frac{l}{\pi a} \int_{0}^{l}\left[\left|\frac{\partial \mathbf{u}}{\partial t}\right|^{2}+\frac{\pi^{2} a^{2}}{l^{2}}|\mathbf{u}|^{2}\right] \mathrm{d} x \\
& \leq \frac{l}{\pi a} E(t), \quad t>0
\end{aligned}
$$


where (10) and the defining equation (4) are used. Also, similarly

$$
0 \leq \delta \int_{0}^{l}|\mathbf{u}|^{2} \mathrm{~d} x \leq \frac{\delta l^{2}}{\pi^{2} a^{2}} \int_{0}^{l} a^{2}\left|\frac{\partial \mathbf{u}}{\partial x}\right|^{2} \mathrm{~d} x \leq \frac{2 \delta l^{2}}{\pi^{2} a^{2}} E(t), \quad t>0 .
$$

Thus using (11) and (12) in eq. (9)

$$
|G(t)| \leq \frac{l}{\pi a}\left(1+\frac{2 \delta l}{\pi a}\right) E(t)=\mu_{0} E(t) \quad(\text { say }), \quad t>0 .
$$

Also from (9),

$$
G(t) \geq \int_{0}^{l} \mathbf{u} \cdot \frac{\partial \mathbf{u}}{\partial t} \mathrm{~d} x \geq-\left|\int_{0}^{l} \mathbf{u} \cdot \frac{\partial \mathbf{u}}{\partial t} \mathrm{~d} x\right| \geq-\frac{l}{\pi a} E(t), \quad t>0 .
$$

The inequalities (13) and (14) yield for $V(t)$ (defined by (8)) the estimates

$$
\left(1-\frac{\varepsilon l}{\pi a}\right) E(t) \leq V(t) \leq\left(1+\varepsilon \mu_{0}\right) E(t), \quad t>0
$$

where we assume that $\varepsilon<\pi a / l$, so that the left hand side of (15) is positive.

Next, differentiating (9) with respect to $t$, and using the governing equation (1b), we obtain

$$
\frac{\mathrm{d} G}{\mathrm{~d} t}=\int_{0}^{l}\left[\mathbf{u} \cdot \frac{\partial^{2} \mathbf{u}}{\partial x^{2}}\left(a^{2}+b \int_{0}^{l}\left|\frac{\partial \mathbf{u}}{\partial x}\right|^{2} \mathrm{~d} x\right)+\left|\frac{\partial \mathbf{u}}{\partial t}\right|^{2}\right] \mathrm{d} x .
$$

Integration by parts of the first term yields

$$
\begin{aligned}
\frac{\mathrm{d} G}{\mathrm{~d} t} & =-\left(a^{2}+b \int_{0}^{l}\left|\frac{\partial \mathbf{u}}{\partial x}\right|^{2} \mathrm{~d} x\right) \int_{0}^{l}\left|\frac{\partial \mathbf{u}}{\partial x}\right|^{2} \mathrm{~d} x+\int_{0}^{l}\left|\frac{\partial \mathbf{u}}{\partial t}\right|^{2} \mathrm{~d} x \\
& \leq-2 E(t)+2 \int_{0}^{l}\left|\frac{\partial \mathbf{u}}{\partial t}\right|^{2} \mathrm{~d} x, \quad t>0 .
\end{aligned}
$$

If we now differentiate (8) with respect to $t$ and then insert the results of (5) and (16), we get

$$
\frac{\mathrm{d} V}{\mathrm{~d} t} \leq 2(\varepsilon-\delta) \int_{0}^{l}\left|\frac{\partial \mathbf{u}}{\partial t}\right|^{2} \mathrm{~d} x-2 \varepsilon E(t), \quad t>0
$$

and so if we choose $\varepsilon<\min \{\delta, \pi a / l\}$,

$$
\frac{\mathrm{d} V}{\mathrm{~d} t} \leq-2 \varepsilon E(t) \leq-\frac{2 \varepsilon}{1+\varepsilon \mu_{0}} V(t), \quad t>0
$$

where (15) has been used. Defining

$$
\mu=\frac{2 \varepsilon}{1+\varepsilon \mu_{0}},
$$


the above inequality becomes

$$
\frac{\mathrm{d} V}{\mathrm{~d} t}+\mu V(t) \leq 0, \quad t>0
$$

and so multiplying by $\exp (\mu t)$ and integrating from 0 to $t$, we obtain

$$
V(t) \leq \mathrm{e}^{-\mu t} V(0), \quad t>0 .
$$

Finally, using (15) again, we obtain the estimate (6) where

$$
M=\frac{1+\varepsilon \mu_{0}}{1-\varepsilon l / \pi a}>1 .
$$

Hence the theorem.

Remark. In the above we have also obtained exponential energy decay estimate for the solution of the nonlinear damped elastic string for initial conditions $\mathbf{u}_{0} \in H^{1}[0, l], \mathbf{u}_{1} \in$ $L^{2}[0, l]$. The decay rate $\mu$ is a function of $\varepsilon$ as given in eq. (17), where $\varepsilon$ is the lesser of the two quantities $\delta$ and $\pi a / l$. Since $\mathrm{d} \mu / \mathrm{d} \varepsilon=2 /\left(1+\varepsilon \mu_{0}\right)^{2}>0, \mu$ is maximum when $\varepsilon$ is maximum. In actual cases $\delta$ is the smaller parameter unless the string is very long. So from the restriction on $\varepsilon$, we can assume $\varepsilon \leq \delta \leq \pi a / l$. Hence $\varepsilon_{\max }=\delta$ and so

$$
\mu_{\max }=\frac{2 \delta}{1+\delta \mu_{0}}=\frac{2 \delta}{1+\frac{\delta l}{\pi a}\left(1+\frac{2 \delta l}{\pi a}\right)} .
$$

The right hand side is a function of $\delta l / \pi a \leq 1$ and is maximum when $\delta l / \pi a=1 / \sqrt{2}$. Hence

$$
\mu_{\max } \leq \frac{2}{1+2 \sqrt{2}} \frac{\pi a}{l}=0.52 \frac{\pi a}{l}
$$

for all possible cases of damping.

It is of some interest to note that Anand [1] in his modal solution of the problem assumed $\mu=\delta$. This assumption holds when $\delta<\pi a / l$. In the contrary case $\mu=\pi a / l$ will suffice exponential decay of the solution.

The quantity $M$ given by eq. (18) however also increases with $\varepsilon$, because $\mathrm{d} M / \mathrm{d} \varepsilon=$ $\left(\mu_{0}+l / \pi a\right) /(1-\varepsilon l / \pi a)^{2}>0$. Thus for $\varepsilon_{\max }=\delta$,

$$
M=\frac{1+\frac{\delta l}{\pi a}\left(1+\frac{2 \delta l}{\pi a}\right)}{1-\delta l / \pi a}=\frac{1+2 \sqrt{2}}{\sqrt{2}-1}=9.3
$$

when $\delta l / \pi a=1 / \sqrt{2}$. Thus if we look for steeply falling estimate, the energy gets multiplied many times in the initial stages.

\section{Quasi-steady amplitude estimate}

Scheefer's inequality (10) when applied to the energy functional $E(t)$ given by equation (4) yields

$$
E(t) \geq \frac{1}{2} \int_{0}^{l}\left[\left|\frac{\partial \mathbf{u}}{\partial t}\right|^{2}+\frac{\pi^{2} a^{2}}{l^{2}}|\mathbf{u}|^{2}\right] \mathrm{d} x+\frac{b}{4} \frac{\pi^{4}}{l^{4}}\left[\int_{0}^{l}|\mathbf{u}|^{2} \mathrm{~d} x\right]^{2}
$$


With the aid of the estimate (6), we obtain

$$
\frac{\pi^{2} a^{2}}{2 l^{2}} \int_{0}^{l}|\mathbf{u}|^{2} \mathrm{~d} x+\frac{b}{4} \frac{\pi^{4}}{l^{4}}\left[\int_{0}^{l}|\mathbf{u}|^{2} \mathrm{~d} x\right] \leq M E(0) \mathrm{e}^{-\mu t}
$$

whose solution yields the estimate for the total amplitude of the string

$$
\int_{0}^{l}|\mathbf{u}|^{2} \mathrm{~d} x \leq \frac{l^{2} a^{2}}{\pi^{2} b}\left[\sqrt{1+\frac{4 b}{a^{4}} M E(0) \mathrm{e}^{-\mu t}}-1\right] .
$$

We say that the estimate (22) is really close for the quasi-steady stage $t \gg 0$ when the velocities die down. This is because the kinetic energy part in the derivation of (22) gets ignored. Apparantly when $t \rightarrow+\infty,|\mathbf{u}| \rightarrow 0$.

\section{Conclusion}

Here we have established uniform stability of a transversely vibrating string (or a metallic wire) fixed at both ends, governed by vector Kirchoff type nonlinear integro-differential equation, which takes into account the string's elasticity and passive viscous damping. It has been proved directly from the equations of motion that the energy of the system decays exponentially with time. Study of nonlinear vibrations assumes significance in analysing slender structural elements capable of withstanding finite deformations. This paper is motivated by such considerations.

\section{Appendix}

Here we give a simple proof of Scheefer's inequality (10). Since $\mathbf{u}(0, t)=\mathbf{0}=\mathbf{u}(l, t)$, we can have a Fourier representation

$$
\mathbf{u}(x, t)=\sum_{n=1}^{\infty} \mathbf{b}_{n}(t) \sin \frac{n \pi x}{l} .
$$

Assuming term-by-term differentiation holds,

$$
\frac{\partial \mathbf{u}}{\partial x}=\frac{\pi}{l} \sum_{n=1}^{\infty} n \mathbf{b}_{n}(t) \cos \frac{n \pi x}{l} .
$$

Applying Parseval's theorem to the two series, we get

$$
\int_{0}^{l}\left|\frac{\partial \mathbf{u}}{\partial x}\right|^{2} \mathrm{~d} x=\frac{l}{2} \frac{\pi^{2}}{l^{2}} \sum_{n=1}^{\infty} n^{2}\left|\mathbf{b}_{n}\right|^{2} \geq \frac{l}{2} \frac{\pi^{2}}{l^{2}} \sum_{n=1}^{\infty}\left|\mathbf{b}_{n}\right|^{2}=\frac{\pi^{2}}{l^{2}} \int_{0}^{l}|\mathbf{u}|^{2} \mathrm{~d} x .
$$

Hence the inequality.

\section{Acknowledgement}

The authors wish to thank the referee for critical comments which led to several clarifications in the text.

\section{References}

[1] Anand G V, Large amplitude damped free vibrations of a stretched string, J. Acoust. Soc. Am. 45 (1969) 1089-1096 
[2] Anand G V, Stability of nonlinear oscillations of stretched strings, J. Acoust. Soc Am. 46 (1969) 667-677

[3] Arosio A and Spagnolo S, Global solution to the Cauchy problem for a nonlinear hyperbolic equation, Nonlin. Partial Diff. Equ. Appl. (eds) H Brezis and J L Lions (1984) (Pitman) vol. VI, pp. 1-26

[4] Gorain G C, Exponential energy decay estimate for the solution of internally damped wave equation in a bounded domain, J. Math. Anal. Appl. 216 (1997) 510-520

[5] Gorain G C and Bose S K, Exact controllability and boundary stabilization of torsional vibrations of an internally damped flexible space structure, J. Optim. Theo. Appl. 99 (1998) 423-442

[6] Gorain G C and Bose S K, Exact controllability and boundary stabilization of flexural vibrations of an internally damped flexible space structure, Appl. Math. Comp. 126 (2002) 341-360

[7] Gough C, The nonlinear free vibration of a damped elastic string, J. Acoust. Soc. Am. 75 (1984) 1770-1776

[8] Hardy G H, Littlewood J E and Polya G, Inequalities (Cambridge University Press) (1964)

[9] Harrison H, Plane and circular motion of a string, J. Acoust. Soc. Am. 20 (1948) 874-875

[10] Kirchoff G, Vorlesungen über Mathematische Physik: Mechanik (Teubner) (1877)

[11] Komornik V, Exact Controllability and Stabilization, the Multiplier Method (Paris: John Wily-Mason) (1994)

[12] Menzala G P, On classical solution of a quasilinear hyperbolic equation, Nonlin. Anal. Theo. Meth. Appl. 3 (1979) 613-627

[13] Mitrinović J, Pečarić J B and Fink A M, Inequalities Involving Functions and their Integrals and Derivatives (Dordrecht: Kluwer) (1991)

[14] Nayfeh A H and Mook D T, Nonlinear Oscillations (New York: John Wiley) (1979)

[15] Newman W G, Global solution of a nonlinear string equation, J. Math. Anal. Appl. 192 (1995) 689-704

[16] Shahruz S M, Bounded-input bounded-output stability of a damped nonlinear string, IEEE Trans. Auto. Contr. 41 (1996) 1179-1182 